\title{
Minus Charge Stimulation Prevents LPS-Induced Liver Injury by Reduction of Nitric Oxide
}

\author{
Fujitoshi Senga', Li Yin ${ }^{1,2}$, Hiroshi Karasuno ${ }^{1,2,3}$, Hirokazu Ohtaki ${ }^{2}$, Tomoya Nakamachi², \\ Kazue Satoh ${ }^{2}$, and Seiji Shioda ${ }^{2, *}$ \\ ${ }^{1}$ ITO Co., LTD., 3-3-3, Toyotama-Minami, Nerima-ku, Tokyo 176-8605, Japan \\ ${ }^{2}$ Department of Anatomy, Showa University School of Medicine, 1-5-8 Hatanodai, Shinagawa-Ku, Tokyo 142-8555, \\ Japan \\ ${ }^{3}$ Koriyama Institute of Health Science, 2-9-3 Zukei, Koriyama-city, Fukushima963-8834, Japan
}

Received 10 October, 2007; Accepted 29 October, 2007

\begin{abstract}
Summary The liver is one of the major target organs affected in sepsis that are usually accompanied with free radical formation. The use of minus charge for the prevention and cure of various radical related diseases is gaining wide importance in the medicinal field. Here, we investigate whether minus charge stimulation (MCS) inhibits nitric oxide (NO) production induced by lipopolysaccharide (LPS) in the mice liver. The survival rate was compared in LPS-treated group with MCS group. The liver NO radical was measured using electron spin resonance technique. Serum alanine transaminase (ALT) was estimated for liver injury. MCS significantly improved the survival rate of LPS-treated mice and inhibited increase of ALT in serum levels. MCS also reduced NO radical production significantly in the LPS-treated mice liver tissue. In conclusion, our results indicate that MCS prevents LPS-induced liver injury, which may be through the inhibition of liver NO radical production.
\end{abstract}

Key Words: minus charge stimulation, liver injury, nitric oxide, electron spin resonance, lipopolysaccharide

\section{Introduction}

Excessive systemic inflammation in sepsis is the most common cause of death in intensive care units [1], ultimately resulting in multiple organ dysfunction syndrome $[2,3]$. The liver plays an important role in the pathogenesis of sepsis both as a source of inflammatory mediators as well as the target organ for the effects of these mediators [4, 5]. Gramnegative bacterial lipopolysaccharide (LPS)-induced lethality is characterized by liver failure, accompanied by severe hepatic injury. This mechanisms is involved in chemical mediators such as superoxide, nitric oxide (NO) radical, and cytokines [6-11]. Thus, LPS is widely used as an

\footnotetext{
*To whom correspondence should be addressed

Tel: +81-3-3784-8103 Fax: +81-3-3784-6815

E-mail: shioda@med.showa-u.ac.jp
}

inducer of various free radicals and cytokines [12].

$\mathrm{NO}$ is a key factor for hepatic injury. NO interacts with superoxide $\left(\mathrm{O}_{2}^{-}\right)$to produce peroxynitrite $\left(\mathrm{ONOO}^{-}\right)$, a potent oxidant [13]. However, the direct measurement of NO radical is not commonly performed because NO has a very short half-life of less than five seconds. For the detection and identification of NO radical, the use of spintrapping compounds appears to be the best method in vivo [14]. By using the electron spin resonance (ESR) spintrapping technique, we have detected in vivo NO radical production in the liver after mice partial hepatectomy [15]. However, it is not yet known the detection of liver NO production after LPS-induced liver injury using ESR technique.

On the other hand, reactive oxygen species (ROS) including NO contribute to the pathogenesis of various acute and chronic liver diseases such as acetaminophen overdose, hemochromatosis, alcoholic liver injury, toxin exposures and viral hepatitis [16-22]. Thus, a treatment of scavenger 
of ROS is expected to prevent these free radical mediated liver diseases. It is well known that electrostatic potential therapy equipment applies small amounts of electrical energy to the entire body and promotes homeostatic functions within the body, such as stimulating blood flow and providing relief from pain and other beneficial effects. Japan is in forefront of development of electrostatic potential equipments for physical therapy. Moreover, the actual results in clinical applications have been accumulated for over 50 years $[23,24]$.

Electrostatic potential therapy is also used to cure headaches, stiff shoulders, insomnia, chronic constipation and various radical related diseases [25, 26]. Furthermore, the use of turning normal water into an antioxidant with minus charge to prevent against superoxide anion radicals and to protect DNA from oxidative damage [27, 28]. However, the mechanisms responsible of electrostatic potential therapy remain poorly understood. The minus charge stimulation (MCS), a kind of electrostatic potential therapy equipments, provides minus charges into body while free radical demands minus charge. Therefore, we hypothesize that MCS may affect on free radical production. In the present study, we examined whether MCS participates in LPS induced hepatic injury in mice as an experimental endotoxic shock model. Moreover, in order to clarify an anti-radical effect of Minus Charge stimulation (MSC), ESR method was used to measure NO free radicals generation directly in the hepatic tissue.

\section{Materials and Methods}

\section{Animals}

Male BALB/c mice ( 8 weeks, weighting 21-26 g) were purchased from Sankyo Labo Service Co. (Tokyo, Japan). Mice were maintained on a $12 \mathrm{~h}$ light/dark cycle at $23 \pm 2{ }^{\circ} \mathrm{C}$ with constant humidity $(45 \pm 10 \%)$. All animal experimental procedures were treated in accordance with guidelines outlined in the Animal Care and Use Committee of Showa University.

The day before the experiment, the animals were anesthetized with sodium pentobarbital, and were gently shaved hair of back about three-square centimeters with a razor to expose skin. Then the animals were returned to their cages and maintained for $24 \mathrm{~h}$.

\section{Minus charge stimulation}

The shaved animals were divided into three groups. One group of the animals $(n=20)$ was injected intraperitoneally (i.p.) with $30 \mathrm{mg} / \mathrm{kg}$ body weight of LPS (Escherichia coli O55:B6, Sigma, Chemical Co., St Louis, MO). The second group of the animals $(n=18)$ which was received the same dose of LPS was immediately applied minus charge stimulation (Ito Co., Ltd, Tokyo, Japan). First, we put the mice into

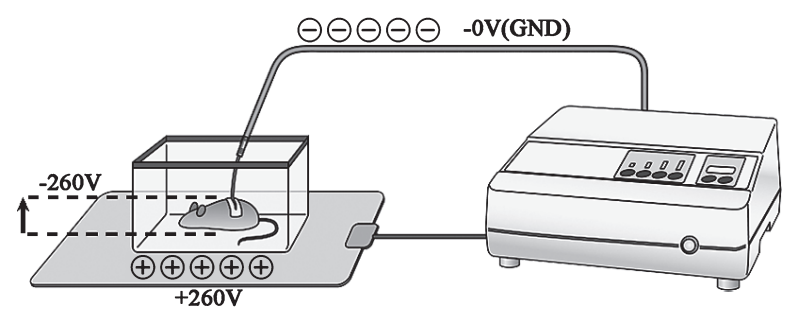

Fig. 1. The diagrams when MCS is applied. The negative pole was fixed on the mice back and was twisted using adhesive tape. Then the plastic cage which mice inside was put onto the insulated sheet which inside is positive pole for the MCS. Finally, the power was turned up to $260 \mathrm{~V}$ and the animals were given free chow and water.

a plastic cage, and the negative pole of MSC was fixed on the mice back with adhesive tape. Then the plastic cage was put onto the insulated sheet which inside is positive pole for the MCS. Finally, the power was turned up to $260 \mathrm{~V}$ and the animals were given free chow and water (Fig. 1). Because the sheet is insulated, it was not able to form a circuit through the mice body. In fact, there was not current flow in MCS equipment, consequently the animals were not given an electric shock. Third group of the animals $(n=20)$ was injected with saline as a sham control. The survival rate was assessed for the next 7 days. The blood samples and liver tissues were obtained from the individual mice at following time point.

\section{NO radical analysis by ESR method}

All the animals were injected with NO trap dosage, $200 \mathrm{mM}$ N-Methyl-D-glucamine dithiocarbamate sodium salt (MGD; Dojin Co., Kumamoto, Japan) and $200 \mathrm{mM}$ ferrous sulfate (FeSO4) complex $(3 ; 1,0.4 \mathrm{ml} / 20 \mathrm{~g}$ mice weight, i.p.) at $30 \mathrm{~min}$ before sacrifice. Once the animals were sacrificed, a piece of remnant right lobe $(100 \mathrm{mg})$ was compressed immediately on a Tissue cell and the level of NO radical was measured by electron spin resonance (ESR) spectroscope (JEOL JES RE1X, X-band, $100 \mathrm{KHz}$ modulation frequency) at room temperature. Instrument settings were as follows: center field, $330 \pm 5.0 \mathrm{mT}$; microwave power, $16 \mathrm{~mW}$; modulation amplitude, $0.5 \mathrm{mT}$; gain, 5000 ; time constant, $1 \mathrm{~s}$; scanning time, $8 \mathrm{~min}$. The ESR signal of the $\left[(\mathrm{MGD})_{2} \mathrm{Fe}\right]-\mathrm{NO}$ complex gave a characteristic 3 peak spectrum, the height of first peak was measured as the ESR intensity. The first peak of 2-Fe(II)-NO radical intensity divided by external standard $\mathrm{Mn}$ value is the NO radical intensity.

To compare the NO radical production in the LPS group and MCS group, we first determine the optimal time point for the single LPS injection in the preliminary experiment. The NO radical intensity was measured at $0,2,4,6$ and $8 \mathrm{~h}$ after LPS injection $(n=6)$. Then, the NO radical intensity 
was measured at optimal time point after LPS treatment in the LPS group and MCS group $(n=6)$.

We also examined whether MCS reduce the NO radical production in vitro. MGD was used as NO trap dosage. 3(2-Hydroxy-1-nitrosohydrazino)-N-methyl-1-propanamine (NOC 7; Dojin Co., Kumamoto, Japan) was used as NO generate dosage. We put the negative pole of MSC into a plastic tube which MGD and NOC 7 inside. Then the plastic tube was put onto the insulated sheet which inside is positive pole for the MCS. Finally, the power was turned up to $260 \mathrm{~V}$. After $5 \mathrm{~min}$, the [(MGD) $\left.{ }_{2} \mathrm{Fe}\right]-\mathrm{NO}$ was measured by ESR.

\section{Serum level of alanine transaminase (ALT) analysis}

At optimal time point after LPS injection, the MCS group and control group $(n=8)$ serum was separated from whole blood by centrifugation at $15,000 \mathrm{rpm}$ for $10 \mathrm{~min}$ at $4^{\circ} \mathrm{C}$, and the serum alanine transaminase (ALT) was measured using Vet Scan Rotar Profiler (ABAXIS, Inc. Union City, CA). This enzyme activity was expressed as an international unit (U/L).

\section{HE staining on Liver tissue}

The control group, LPS and MCS group $(n=3)$ mouse were anesthetized with barbiturate at $0,2,4$ and $6 \mathrm{~h}$ after LPS injection and they were perfused transcardially with $2 \%$ paraformaldehyde solution in $0.1 \mathrm{M}$ phosphate buffer. The liver tissues were immersed for 2 days in a solution containing $20 \%$ sucrose for cytoprotection, and they were embedded in Tissue Tek OCT compound (Miles Laboratories, Inc. Naperwille, IL). The frozen sections of 8 - $\mu \mathrm{m}$ thickness were prepared. The histology change was compared in liver tissue by hematoxylen eosin staining.

\section{Results}

\section{Effects of MCS on animal survival rate}

All animals $(n=20)$ died from $12 \mathrm{~h}$ till $24 \mathrm{~h}$ with only LPS treatment. In contrast, the MCS treatment after LPS

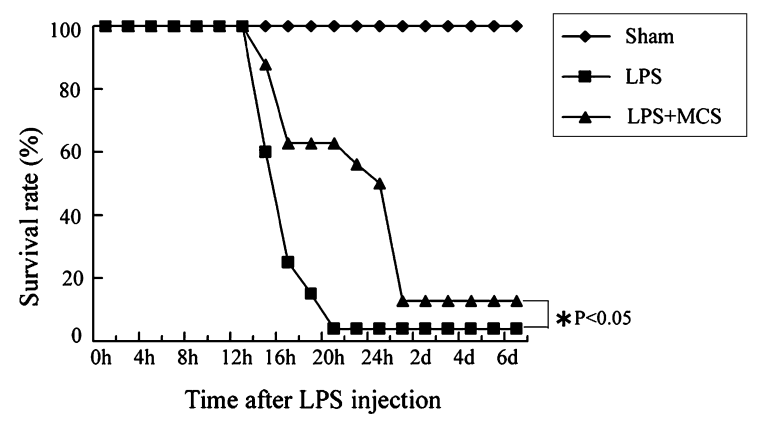

Fig. 2. Effect of MCS on the survival rate of mice after LPS injection. Mice were treated with LPS and saline $(30 \mathrm{mg}$ / kg, i.p.) as described above. MCS was administrated immediately after LPS injection. MCS significantly improved the mice survival rate of from $12 \mathrm{~h}$ to 2 days after LPS treated. $t$ test $p<0.05$ MCS group as compared with LPS group $(n=20)$.
A

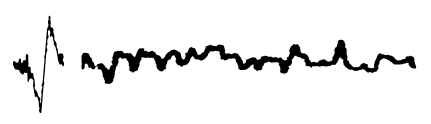

B

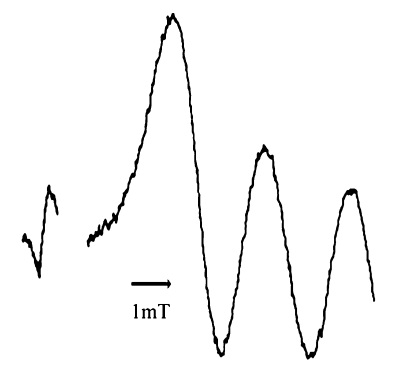

C

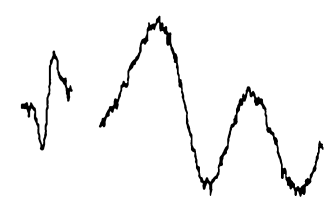

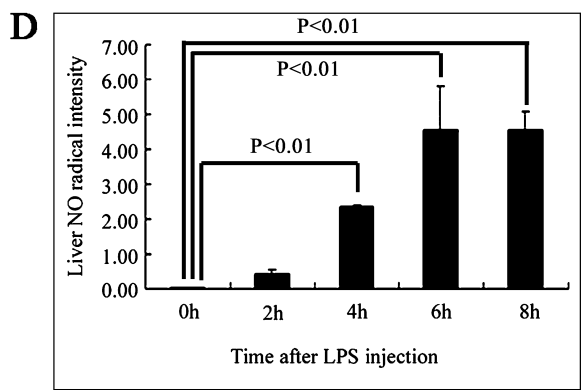

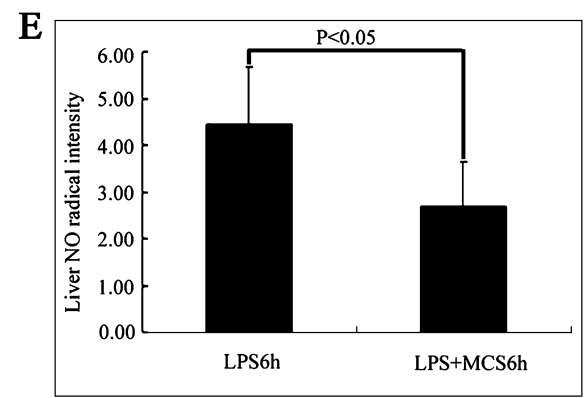

Fig. 3. ESR spectra of the $\left[(\mathrm{MGD})_{2} \mathrm{Fe}\right]-\mathrm{NO}$ complex in the circulation of the mice liver after LPS administration. No signal of NO radical was detected in the control mice liver (A). A weak ESR signal of the [(MGD) $\left.{ }_{2} \mathrm{Fe}\right]-\mathrm{NO}$ complex was detected when the mouse was treated by MCS compared (C) with only LPS injection (B) at $6 \mathrm{~h}$. Bar graphs showing NO radical intensity induced by LPS injection in the liver tissue at each time point and peaked at $6 \mathrm{~h}$ after LPS injection (D) $t$ test $p<0.01$. MCS prevented mice liver NO radical production at $6 \mathrm{~h}$ after LPS injection (E). Values are expressed as the mean \pm SE for each group $(n=6)$, $t$ test $p<0.05$. 
administration improved the mortality from $60 \%$ to $40 \%$ at $16 \mathrm{~h}$ till 2 days (Fig. 2). These results showed clearly that MCS treatment had an effect on the rate of survival from LPS administration in mice.

\section{The intensity of $\mathrm{NO}$ radical in the liver}

In control, when the animals were injected with $200 \mathrm{mM}$ N-Methyl-D-glucamine dithiocarbamate sodium salt and $200 \mathrm{mM}$ ferrous sulfate $\left(\mathrm{FeSO}_{4}\right)$ complex without a prior LPS injection, characteristic line of ESR spectrum was not detected in the liver (Fig. 3A). At $6 \mathrm{~h}$ prior treatment with LPS group, the [(MGD) $\left.)_{2} \mathrm{Fe}\right]-\mathrm{NO}$ complex gave a characteristic 3 peaks spectrum. The height of first peak spectrum was $4.4 \pm 1.2 \mathrm{~mm}$ (Fig. 3B). However NO radical ESR spectrum was significantly inhibited to $2.7 \pm 1.0 \mathrm{~mm}$ in the MCS group at the same time point (Fig. 3C).

Then the height of first peak divided by external standard Mn value was measured as the ESR intensity. We measured the time-course changes of NO radical intensity after LPS administration. The intensity of NO radical was gradually increased at $2 \mathrm{~h}$ after LPS administration. At $4 \mathrm{~h}$ after LPS injection, the intensity of NO was rapidly increased. Significant increase of NO radical intensity was measured at $6 \mathrm{~h}$ and kept till $8 \mathrm{~h}$ after LPS injection $(p<0.05)$ (Fig. 3D). Then we compared the NO radical intensity at $6 \mathrm{~h}$ after LPS injection in the LPS group and MCS group. Expectedly, MCS significantly inhibited NO radical intensity at the same time point $(p<0.05)$ (Fig. 3E). These data demonstrate that MCS prevents NO radical production induced with LPS injection.

For the in vitro experiment, a strong three-line ESR spectrum of [(MGD) $\left.{ }_{2} \mathrm{Fe}\right]-\mathrm{NO}$ complex was observed at 2 min after NOC7 mixed with spin-trapping, the (MGD) $)_{2} \mathrm{Fe}$ complex (Fig. 4A). The first peak divided by external standard Mn value was measured as the ESR intensity. A weak ESR signal of [(MGD) $\left.{ }_{2} \mathrm{Fe}\right]-\mathrm{NO}$ complex was detected when MCS was applied 5 min (Fig. 4B). Then we compared the NO radical intensity in control and MCS group. Expectedly, MCS also significantly inhibited NO radical intensity in vivo $(p<0.01)$ (Fig. 4C).

\section{Effect of MCS of serum level of ALT}

The serum values of ALT were analyzed to evaluate liver injury. The LPS-treatment increased serum values of ALT in mice. The increase in ALT value was reduced significantly by administration of MCS at $6 \mathrm{~h}$ after LPS injection (Fig. 5).

\section{Histological observation}

The liver tissue was compared in LPS and MCS group. At $0 \mathrm{~h}$ after LPS injection, the morphology of liver is normal in the LPS or MCS group (Fig. 6A, B). No morphological differences were observed in the LPS and MCS group at $2 \mathrm{~h}$ after LPS injection (Fig. 6C, D). Many irregular damaged hepatocytes were observed in the LPS group at $6 \mathrm{~h}$,

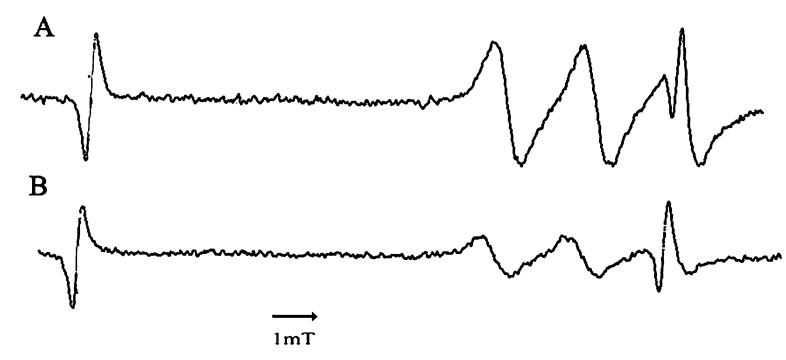

C

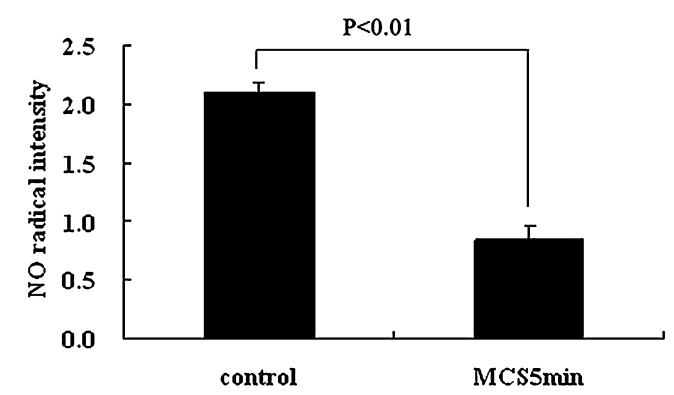

Fig. 4. ESR spectra of the [(MGD) $\left.)_{2} \mathrm{Fe}\right]-\mathrm{NO}$ complex in vitro at 2 min after NOC7 mixed with (MGD) ${ }_{2} \mathrm{Fe}$ complex. ESR signal of the $\left[(\mathrm{MGD})_{2} \mathrm{Fe}\right]-\mathrm{NO}$ complex was detected in the control (A) and 5 min MCS-treated group (B). Bar graphs showing MCS inhibited NO radical intensity compared with control (C). Values are expressed as the mean \pm SE for each group, $t$ test $p<0.01$.

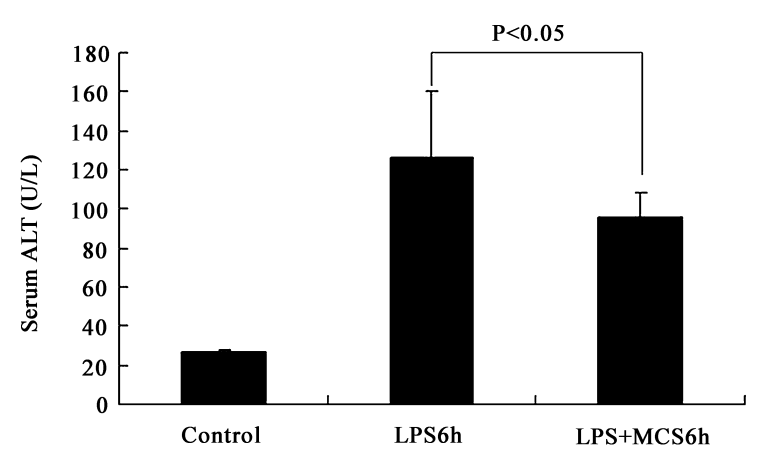

Fig. 5. The effect of MCS of serum level of ALT. The increases in serum ALT value were reduced significantly by administration of MCS at $6 \mathrm{~h}$ after LPS injection. Serum ALT are shown as mean $\pm \mathrm{SE}(n=6), t$ test $p<0.05$.

and the MCS inhibited the hepatocytes damage clearly (Fig. 6E, F).

\section{Discussion}

The present study demonstrates that MCS treatment is effective in protecting mice from liver injury due to LPS. The survival rate of mice was $0 \%$ at $24 \mathrm{~h}$ after LPS injection. 

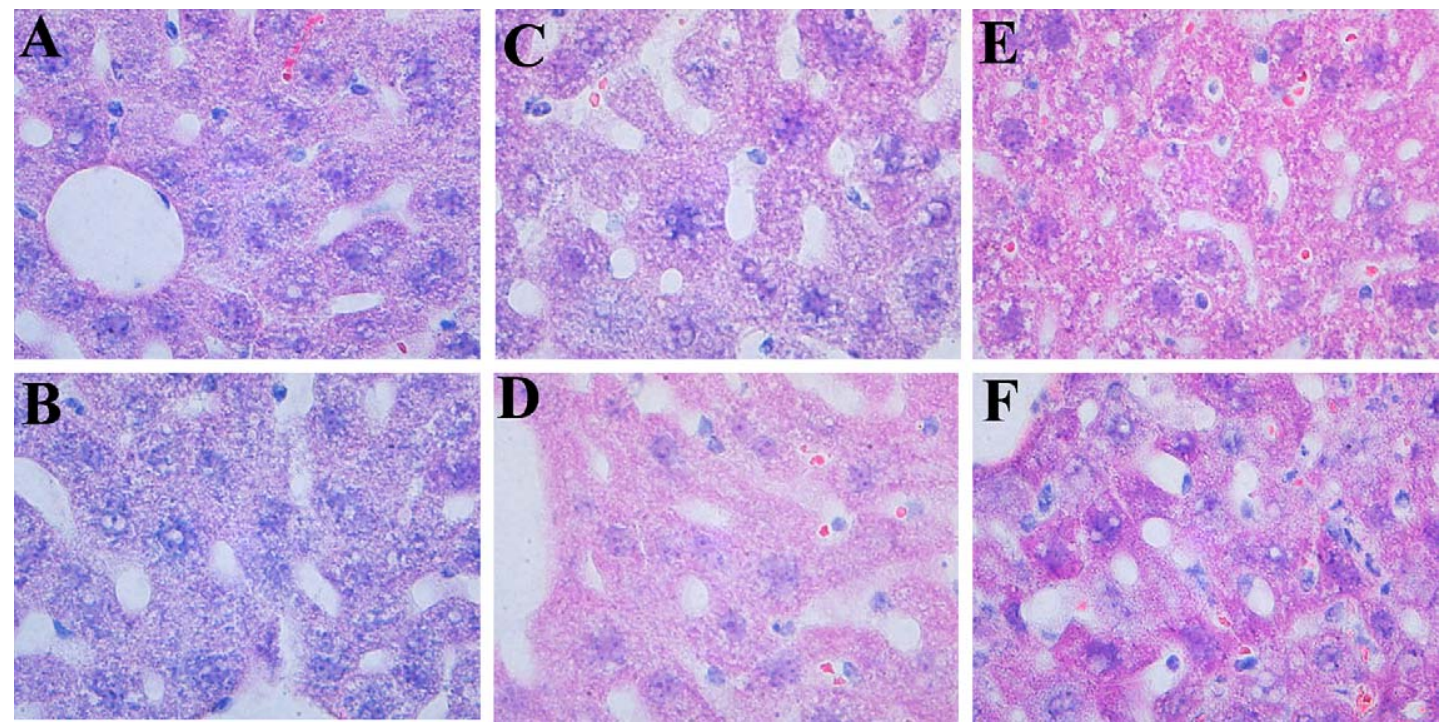

Fig. 6. Liver HE staining. The liver tissue was stained at 0, 2 and $6 \mathrm{~h}$ after LPS (A, C, E) and MCS group (B, D, F).

In contrast, MCS treatment markedly improved the survival rate of the animals. LPS administration caused severe hepatic dysfunction, as revealed by marked increase in serum ALT. However, MCS treatment markedly improved the LPS-induced hepatic dysfunction, as shown a significant reduction of serum ALT enzyme activity, and the appearance of pathological changes, resulting in an improvement in the survival rate of the animal. These results demonstrate that MCS prevents LPS-induced hepatocytes cellular damage that occurs as a result of hepatic injury and subsequent injury to multiple organs.

It has been demonstrated in many animal models in vivo and in vitro that LPS induces the overproduction of ROS, including free radical such as NO radical [12, 22, 29]. The liver is one organ that is clearly influenced by NO. Consequently, oxidative damage occurs in the liver [4, 19]. The balance of NO and ROS is probably also important in regulating the biological functions of NO. A low dose of NO servers to maximize blood perfusion, prevent neutralize toxic oxygen radicals in the liver during acute sepsis. NO also demonstrates antimicrobial and anti-apoptosis properties during hepatitis infection and other inflammatory processes. However, when a large sustained amount of NO is present, NO might become genotoxic and lead to the development of liver injury [4]. In the present study, we first measured directly NO radical production in the liver tissue after LPS injection using ESR method. NO radical increased gradually at $2 \mathrm{~h}$ and increased rapidly at $4 \mathrm{~h}$ after LPS injection. Finally, the intensity of NO radical reached a maximum after $6 \mathrm{~h}$. We considered this acute and mass-produced increasing of NO is harmful to the liver tissue. Expectedly, MCS treatment inhibited significantly at $6 \mathrm{~h}$ LPS-induced NO production increasing in the mice. MCS may thus prevent harmful effect of NO to the liver injury induced by LPS injection.

We also measured ALT in serum level, another general marker of liver injury. The level of ALT was increased at $6 \mathrm{~h}$ after LPS injection. Expectedly, MCS treatment significantly reduced ALT level after LPS injection. Taken together, MCS could protect hepatic injury induced by LPS treatment. The liver is one of the most important body organs in that it performs so many different functions. The liver makes proteins, eliminates waste material form the body and so on. Therefore, the serious liver injury may induce excessive systemic inflammation in sepsis, which is the most common cause of death in intensive care units [1]. This is may be explain that MCS increased the survival rate of mice after LPS injection.

In conclusion, MCS prevents LPS-induced liver injury, which may be through the inhibition of NO radical production in the liver tissue. The minus charge exists in the nature, and the MCS produces no harmful side effects to the body. Therefore, MCS may be a safe and effective therapy for the many liver diseases that companied with NO radical production in the future. It is also necessary to study the mechanism of MCS protecting on hepatic injury in the future.

\section{Acknowledgments}

This study was supported in part by Showa University High-Technology Research Center Project from the Ministry of Education, Science, Sports and Culture of Japan and Research on Health Sciences focusing on Drug Innovation from The Japan Health Sciences Foundation. 


\section{Abbreviations}

ESR, electron spin resonance; LPS, lipopolysaccharide; MCS, minus charge stimulation; NO, nitric oxide; PBS, phosphate buffer saline; ROS, reactive oxygen species; ALT, alanine transaminase.

\section{References}

[1] Johnson, D. and Mayers, I.: Multiple organ dysfunction syndrome: a narrative review. Can. J. Anaesth., 48, 502-509, 2001.

[2] Baue, A.E., Durham, R., and Faist, E.: Systemic inflammatory response syndrome (SIRS), multiple organ dysfunction syndrome (MODS), multiple organ failure (MOF): are we winning the battle? Shock, 10, 79-89, 1998.

[3] Cohen, J.: The immunopathogenesis of sepsis. Nature, 420, 885-891, 2002.

[4] Hon, W.M., Lee, K.H., and Khoo, H.E.: Nitric oxide in liver diseases: Friend, Foe, or Just Passerby? Ann. NY. ACAD. SCI., 962, 275-295, 2002.

[5] Szabo, G., Romics, L.J., and Frendl, G..: Liver in sepsis and systemic inflammatory response syndrome. Clin. Liver Dis., 6, 1045-1066, 2002.

[6] Bykov, I., Ylipaasto, P., Eerola, L., and Lindros, K.O.: Phagocytosis and LPS-stimulated production of cytokines and prostaglandin E2 is different in Kupffer cells isolated from the periportal or perivenous liver region. Scand. J. Gastroenterol., 38, 1256-1261, 2003.

[7] Enomoto, N., Takei, Y., Hirose, M., Ikejima, K., and Miwa, H.: Estriol enhances lipopolysaccharide-induced increases in nitric oxide production by Kupffer cells via mechanisms dependent on endotoxin. Alcohol. Clin. Exp. Res., 26, 66S69S, 2002.

[8] Hewett, J.A. and Roth, R.A.: Hepatic and extrahepatic pathobiology of bacterial lipopolysaccharides. Pharmacol. Rev., 45, 382-411, 1993.

[9] Suzuki, S., Nakamura, S., Serizawa, A., Sakaquchi, T., Konno, H., Muro, H., Kosuqi, I., and Baba, S.: Role of Kupffer cells and the spleen in modulation of endotoxininduced liver injury after partial hepatectomy. Hepatology, 24, 219-225, 1996.

[10] Tsukada, S., Enomoto, N., Takei, Y., Hirose, M., Ikejima, K., Kitamura, T., and Sato, N.: Dalteparin sodium prevents liver injury due to lipopolysaccharide in rat through suppression of tumor necrosis factor-alpha production by Kupffer cells. Alcohol. Clin. Exp. Res., 27, 7S-11S, 2003.

[11] Wang, J.H., Redmond, H.P., Watson, R.W., and Bouchier, H.D.: Role of lipopolysaccharide and tumor necrosis factoralpha in induction of hepatocyte necrosis. Am. J. Physiol., 269, G297-304, 1995.

[12] Mayeux, P.R.: Pathobiology of lipopolysaccharide. J. Toxicl. Environ. Health., 51, 415-435, 1997.

[13] Beckman, J.S. and Koppenol, W.H.: Nitric oxide, super oxide, and peroxynitrite: the good, the bad, and the ugly. Am. J. Physiol., 271, 1424-1437, 1996.

[14] Nakai, K., Kadiiska, M.B., Jiang, J.J., Stadler, K., and Mason, R.P.: Free radical production requires both inducible nitric oxide synthase and xanthine oxidase in LPS-treated skin. PNAS., 103, 4616-4621, 2006.

[15] Mitamura, K., Otsuka, K., Kato, H., Enami, Y., Murai, N., Niiya, T., Aoki, T., Shimizu, Y., Satoh, K., Tobe, T., Shioda, S., and Kusano, M.: Detection of nitric oxide generation by electron spin resonance spectroscopy after partial hepatectomy. Showa Univ. J. Med. Sci., 15, 237-244, 2003.

[16] Bacon, B.R., Tavill, A.T., Brittenham, G.M., Park, C.H., and Recknagel, R.O.: Hepatic lipid peroxidation in vivo in rats with chronic iron overload. J. Clin. Invest., 71, 429-439, 1983.

[17] Kyle, M.E., Miccadei, S., Nakae, D., and Farber, J.L.: Superoxide dismutase and catalase protect cultured hepatocytes from the cytotoxicity of acetaminophen. Biochem. Biophys. Res. Commun., 149, 889-896, 1987.

[18] Machilin, L.J. and Bendich, A.: Free radical tissue damage: protective role of antioxidant nutrients. FSAEB. J., 1, 441445, 1987.

[19] Mayer, M., Caselman, W.H., Schulter, V., Schreck, R., Hofschneider, P.H., and Baeuerle, P.A.: Hepatitis B virus transactivator MHBskB, selective inhibition of antioxidants and integral membrane localization. EMBO. J., 11, 29912998, 1993.

[20] Shaw, S., Jayatilleke, E., Ross, W.A., Gordon, E., and Lieber, C.S.: Ethanol induced lipid peroxidation: potentiation by long-term alcohol feeding and attenuation by methionine. $J$. Lab. Clin. Med., 98, 417-425, 1981.

[21] Slater, T.F.: Free radical mechanisms in tissue injury. Biochem. J., 222, 1-15, 1984.

[22] Hong, J.Y., Sato, E.F., Hiramoto, K., Nishikawa, M., and Inoue, M.: Mechanism of liver injury during obstructive jaundice: role of nitric oxide, splenic cytokines, and intestinal flora. J. Clin. Biochem. Nutr., 40, 184-193, 2007.

[23] Suzuki, S.: Electrical resistively of rat myocardial tissue and its change during postmatal growth. Tohoku J. Exp Med., 9, 194-201, 1971.

[24] Ito, F. and Furuya, K.: The effect of high voltage alternating current upon a human body the change endocrine system and serum lipids. J. JA. Phys. M. Baln. Clim., 45, 6-17, 1981.

[25] Hara, H.: On the effect of ac. Electrostatic high voltage potential load upon the blood electrolytes. Niigata Med. J., 75, 265-273, 1961.

[26] Hashimoto, T.: The effect of static electric field caused by high voltage alternating current on callus formation. J. Kyoto Pref. Univ. Med., 84, 89-108, 1975.

[27] Hanaoka, K., Sun, D., Lawrence, R., Kamitani, Y., and Fernandes, G.: The mechanism of the enhanced antioxidant effects against superoxide anion radicals of reduced water produced by electrolysis. Biophys. Chem., 107, 71-82, 2004.

[28] Shirahata, S., Kabayama, S., Nakano, M., Miura, T., Kusumoto, K., Gotoh, M., Hayashi, H., Otsubo, K., Morisawa, S., and Katakura, Y.: Electrolyzed-reduced water scavenges active oxygen species and protects DNA from oxidative damage. Biochem. Biophys. Res. Commun., 234, 269-274, 1997.

[29] Hon, W.M., Lee, K.H., and Khoo, H.E.: Nitric oxide in liver diseases: friend, foe, or just passerby? Ann. N. Y. Acad. Sci., 962, 275-295, 2002. 